\title{
Etidronate Disodium
}

National Cancer Institute

\section{Source}

National Cancer Institute. Etidronate Disodium. NCI Thesaurus. Code C831.

A synthetic therapeutic diphosphonate analogue of endogenous pyrophosphate. As a member of the family of drugs known as bisphosphonates, etidronate disodium differs from endogenous pyrophosphate in its resistance to enzymatic hydrolysis. This agent adsorbs to hydroxyapatite cells and reduces the number of osteoclasts, thereby inhibiting abnormal bone resorption. Etidronate may also directly stimulate bone formation by osteoblasts. ( $\mathrm{NCl} 04)$ 